\title{
Muito Baixo Peso Ponderal em Pacientes Tratados com Angioplastia Coronária: Impacto na Mortalidade Precoce e Tardia
}

\author{
Pedro A. Lemos ${ }^{1}$, Expedito E. Ribeiro', Luiz J. Kajita', Antonio Esteves Filho', Carlos A. H. Campos', \\ Breno A. A. Falcão', Marco A. Perin'1, Marcus N. da Gama'1, Pedro E. Horta', Gilberto G. Marchiori', \\ Andre G. Spadaro, Paulo R. Soares ${ }^{1}$, Sílvio Zalc1 ${ }^{1}$, Eulógio E. Martinez ${ }^{1}$
}

\section{RESUMO}

Fundamentos: Pacientes de muito baixo peso tratados com intervenção coronária percutânea têm maior risco de complicações durante a internação. Até o momento, não existem estudos para avaliar o efeito a longo prazo do baixo peso após angioplastia coronária na população brasileira. Método: Um total de 3.687 pacientes foi separado em dois grupos, de acordo com o índice de massa corporal (IMC), calculado como peso (em quilogramas) dividido pela altura (em metros) ao quadrado: grupo baixo peso (IMC $\leq 20 \mathrm{~kg} / \mathrm{m}^{2} ; 125$ pacientes) e grupo não-baixo peso (IMC > $20 \mathrm{~kg} / \mathrm{m}^{2}, 3.562$ pacientes). A mortalidade intrahospitalar foi avaliada prospectivamente durante a internação inicial. Após a alta, a ocorrência de óbito foi acessada por meio da revisão dos registros hospitalares e contato telefônico. Resultados: Pacientes com IMC $\leq 20 \mathrm{~kg} / \mathrm{m}^{2}$ apresentavam peso, altura e IMC médios de 49,4 $\pm 7,1 \mathrm{~kg}$, $1,62 \pm 0,10 \mathrm{~m}$, e $18,7 \pm 1,1 \mathrm{~kg} / \mathrm{m}^{2}$, respectivamente. O peso, a altura e o IMC de pacientes com IMC > $20 \mathrm{~kg} / \mathrm{m}^{2}$ foram de 74,4 $\pm 13,8 \mathrm{~kg}, 1,64 \pm 0,09 \mathrm{~m}$, e $27,3 \pm 4,3 \mathrm{~kg} / \mathrm{m}^{2}$, respectivamente ( $p<0,01$ para todas as características). Pacientes do grupo baixo peso apresentaram mortalidade significativamente maior que pacientes com IMC > $20 \mathrm{~kg} / \mathrm{m}^{2}$ após 2,5 anos da angioplastia $(19,4 \%$ vs. $6,9 \%$, respectivamente; hazard ratio [HR]: 2,51, intervalo de confiança de 95\% [IC 95\%]: 1,61-3,91; p < 0,01). Após o ajuste multivariado para a presença de outros fatores de risco, a presença de $\mathrm{IMC} \leq 20 \mathrm{~kg} / \mathrm{m}^{2}$ persistiu como fator independente associado a aumento da mortalidade (HR: 2,04; IC 95\%: 1,28-3,25; $p<0,01)$. Conclusões: $O$ muito baixo peso ponderal, indicado pelo $I M C \leq 20 \mathrm{~kg} / \mathrm{m}^{2}$, é um fator de risco independente para a ocorrência de óbito precoce e tardio nos primeiros 2,5 anos após intervenção coronária percutânea. Pela facilidade de sua identificação, a pes-

\section{SUMMARY}

Very Low Ponderal Weight in Patients Treated with Coronary Angioplasty: Impact on Early and Late Mortality

Background: Patients with very low weight treated with percutaneous coronary intervention have a greater risk of complications during hospitalization. So far, there have been no studies to evaluate the long-term effect of low weight after coronary angioplasty in the Brazilian population. Methods: A total of 3,687 patients were divided into two groups according to their body mass index (BMI), calculated by dividing the weight in kilograms by the height in metres squared: low-weight group (BMI $<20 \mathrm{~kg} / \mathrm{m}^{2} ; 125$ patients), and non-low-weight group (BMI > $20 \mathrm{~kg} / \mathrm{m}^{2}$; 3,562 patients). The in-hospital mortality was evaluated prospectively during first admission. After discharge, death occurrence was assessed by reviewing hospital records and through telephone contact. Results: Patients with BMI $\leq 20 \mathrm{~kg} / \mathrm{m}^{2}$ presented weight, height, and BMI averages of $49.4 \pm 7.1 \mathrm{~kg}, 1.62 \pm 0.10 \mathrm{~m}$, and $18.7 \pm 1.1 \mathrm{~kg} / \mathrm{m}^{2}$, respectively. The weight, height and $\mathrm{BMI}$ of patients with $\mathrm{BMI}>20 \mathrm{~kg} / \mathrm{m}^{2}$ was $74.4 \pm 13.8 \mathrm{~kg}, 1.64 \pm 0.09 \mathrm{~m}$, and $27.3 \pm 4.3 \mathrm{~kg} / \mathrm{m}^{2}$, respectively $(p<0.01$ for all characteristics). Patients from the low-weight group showed significantly higher mortality than patients with BMI > $20 \mathrm{~kg} / \mathrm{m}^{2}$ 2.5 years after angioplasty $(19.4 \%$ vs. $6.9 \%$, respectively; hazard ratio $[H R]: 2.51 ; 95 \%$ confidence interval $[95 \%$ $\mathrm{Cl}$ : $1.61-3.91 ; \mathrm{p}<0.01)$. After multivariate adjustment for other risk factors, the presence of $\mathrm{BMI} \leq 20 \mathrm{~kg} / \mathrm{m}^{2}$ persisted as an independent factor associated to increased mortality (HR: 2.04; 95\% Cl: 1.28-3.25; $\mathrm{p}<0.01$ ). Conclusions: The very low ponderal weight, indicated by a body mass index $\leq 20 \mathrm{~kg} / \mathrm{m}^{2}$, is an independent risk factor for early

1 Instituto do Coração do Hospital das Clínicas da Faculdade de Medicina da Universidade de São Paulo (InCor/HC-FMUSP) - São Paulo, SP.

Correspondência: Pedro A. Lemos. Av. Dr. Enéas Carvalho de Aguiar, 44 - Bloco I - 3o andar - São Paulo, SP - CEP 05403-000 E-mail: pedro.lemos@incor.usp.br

Recebido em: 15/7/2008 • Aceito em: 30/10/2008 
quisa do muito baixo peso pode ser amplamente utilizada como ferramenta prognóstica para pacientes tratados com angioplastia no dia-a-dia.

DESCRITORES: Angioplastia transluminal percutânea coronária. Índice de massa corporal. Prognóstico.

$\mathbf{N}$ os últimos anos, diversos estudos têm avaliado o impacto do peso corpóreo sobre a prevalência de doença cardiovascular e o risco de complicações futuras durante a evolução ${ }^{1-8}$. Mundialmente, forte ênfase tem sido dada aos efeitos deletérios da obesidade $^{1-9}$, cuja freqüência tem aumentando progressivamente em países ocidentais. No entanto, pouca informação científica está atualmente disponível sobre pacientes com muito baixo peso ponderal e o impacto dessa característica sobre o prognóstico de indivíduos com ou sem doença coronariana.

Evidências prévias sugerem que esse subgrupo apresenta maior risco para eventos adversos ${ }^{2,4,9,10}$. Em particular, pacientes coronarianos de muito baixo peso tratados com intervenção percutânea têm demonstrado maior propensão a complicações fatais e não-fatais durante a internação ${ }^{4,9,10}$. No entanto, não existem estudos dedicados a avaliar o efeito a longo prazo do baixo peso em pacientes com coronariopatia obstrutiva, especialmente na população brasileira.

O presente estudo objetiva, portanto, avaliar o impacto do baixo peso ponderal sobre a mortalidade tardia de pacientes brasileiros tratados com angioplastia coronária.

\section{MÉTODO}

\section{População e definições}

De setembro de 1998 a dezembro de 2003, um total de 4.289 pacientes consecutivos, moradores do Estado de São Paulo, foi tratado pelo Sistema Único de Saúde (SUS) brasileiro com intervenção coronária percutânea em nossa instituição. Os dados de peso e altura estavam disponíveis no momento da angioplastia para um total de 3.687 (86\%) pacientes, os quais compõem a população do presente estudo.

Os pacientes incluídos foram divididos em dois grupos, de acordo com o índice de massa corporal (IMC), calculado como peso (em quilogramas) dividido pela altura (em metros) ao quadrado:

- grupo baixo peso (IMC $\leq 20 \mathrm{~kg} / \mathrm{m}^{2} ; 125$ pacientes); pacientes).

- grupo não-baixo peso $\left(\mathrm{IMC}>20 \mathrm{~kg} / \mathrm{m}^{2} ; 3.562\right.$

Características basais e do procedimento foram coletadas prospectivamente para todos os pacientes and late death in the first 2.5 years after percutaneous intervention. Due to its easy identification, very low weight can be widely used as a predictive tool for patients routinely treated with angioplasty.

DESCRIPTORS: Angioplasty, transluminal, percutaneous coronary. Body mass index. Prognosis.

em base de dados eletrônica. Angiografia quantitativa offline foi obtida para todas as lesões, utilizando um sistema computadorizado previamente validado (CASS II, Pie Medical, Maastricht, Holanda) ${ }^{11}$.

A estratégia de tratamento foi definida pelo hemodinamicista responsável pelo caso e seguiu diretrizes de tratamento ótimo atual ${ }^{12}$. Somente stents nãofarmacológicos eram disponíveis no período de inclusão. Ticlopidina ou clopidogrel foram administrados por um mês após o procedimento índice.

\section{Seguimento clínico}

Dados sobre a mortalidade intra-hospitalar foram coletados prospectivamente durante a internação inicial. Após a alta, os pacientes foram avaliados periodicamente em nossa clínica ambulatorial. A ocorrência de óbito foi acessada pela revisão de todas as passagens dos pacientes em nossa instituição e afiliadas, além de contato telefônico quando necessário.

\section{Análise estatística}

Variáveis categóricas foram apresentadas como porcentagem e comparadas por meio do teste exato de Fisher. Variáveis contínuas foram apresentadas por suas médias e desvios padrão. A incidência cumulativa de óbito foi estimada pelo método de KaplanMeier. Modelos de univariados e multivariados utilizando a regressão de Cox foram utilizados para identificar a relação entre possíveis preditores basais e mortalidade durante o seguimento clínico. O impacto do baixo peso ponderal sobre a ocorrência de eventos fatais foi avaliado após análise multivariada para os seguintes parâmetros, tipicamente relacionados a pior prognóstico: idade, diabetes melito, infarto do miocárdio prévio, insuficiência cardíaca, infarto agudo do miocárdio à admissão, cirurgia de revascularização do miocárdio prévia, doença coronariana triarterial, insuficiência renal crônica dialítica, doença vascular periférica e acidente vascular cerebral prévio.

\section{RESULTADOS}

Pacientes do grupo baixo peso apresentaram diferenças significativas em suas características basais e do procedimento quando comparados a pacientes com IMC > $20 \mathrm{~kg} / \mathrm{m}^{2}$ (Tabela 1). Em especial, pacientes com baixo IMC eram mais freqüentemente do sexo femini- 
TABELA 1

Características basais e do procedimento de pacientes do grupo baixo peso (índice de massa corporal $\leq 20 \mathrm{~kg} / \mathrm{m}^{2}$ ) e do grupo não-baixo peso (índice de massa corporal $>20 \mathrm{~kg} / \mathrm{m}^{2}$ )

\begin{tabular}{|c|c|c|c|}
\hline & $\begin{array}{l}\text { Grupo não-baixo peso } \\
(n=3.562)\end{array}$ & $\begin{array}{l}\text { Grupo baixo peso } \\
\quad(n=125)\end{array}$ & $\mathbf{p}$ \\
\hline Sexo masculino & 67,3 & 53,6 & $<0,01$ \\
\hline Idade, anos & $61,9 \pm 11,4$ & $65,6 \pm 13,3$ & $<0,01$ \\
\hline Peso, kg & $74,4 \pm 13,8$ & $49,4 \pm 7,1$ & $<0,01$ \\
\hline Altura, $\mathrm{m}$ & $1,64 \pm 0,09$ & $1,62 \pm 0,10$ & $<0,01$ \\
\hline Índice de massa corporal, kg/m² & $27,3 \pm 4,3$ & $18,7 \pm 1,1$ & $<0,01$ \\
\hline Diabetes melito & 27,6 & 16,8 & 0,03 \\
\hline Não-insulino-dependente & 22,4 & 12,8 & \\
\hline Insulino-dependente & 5,2 & 4,0 & \\
\hline Hipertensão & 72,1 & 66,4 & 0,2 \\
\hline Hipercolesterolemia & 52,5 & 39,2 & $<0,01$ \\
\hline Tabagismo atual & 21,2 & 28,0 & 0,08 \\
\hline Acidente vascular cerebral prévio & 3,0 & 6,4 & 0,06 \\
\hline Insuficiência renal crônica dialítica & 0,8 & 4,8 & $<0,01$ \\
\hline Doença vascular periférica & 2,8 & 5,6 & 0,09 \\
\hline Infarto do miocárdio prévio & 32,3 & 38,4 & 0,2 \\
\hline Insuficiência cardíaca & 13,3 & 19,2 & 0,06 \\
\hline Angioplastia coronária prévia & 11,8 & 10,4 & 0,8 \\
\hline Cirurgia de revascularização miocárdica prévia & 14,7 & 13,6 & 0,8 \\
\hline Infarto agudo do miocárdio à admissão & 32,7 & 28,0 & 0,3 \\
\hline Padrão coronário & & & 0,04 \\
\hline Uniarterial & 41,3 & 51,2 & \\
\hline Biarterial & 32,5 & 22,8 & \\
\hline Triarterial & 26,2 & 26,0 & \\
\hline \multicolumn{4}{|l|}{ Vaso-alvo } \\
\hline Tronco da coronária esquerda & 1,2 & 1,6 & 0,7 \\
\hline Artéria descendente anterior & 48,0 & 46,4 & 0,8 \\
\hline Artéria circunflexa & 27,1 & 22,4 & 0,3 \\
\hline Artéria coronária direita & 32,2 & 28,0 & 0,4 \\
\hline Enxerto & 6,0 & 10,4 & 0,06 \\
\hline Número de lesões tratadas & $1,4 \pm 0,7$ & $1,4 \pm 0,8$ & 0,6 \\
\hline Número de stents implantados & $1,2 \pm 0,7$ & $1,3 \pm 0,7$ & 0,4 \\
\hline Diâmetro de referência, mm & $2,62 \pm 0,52$ & $2,52 \pm 0,49$ & 0,02 \\
\hline Diâmetro luminal mínimo pré-procedimento, mm & $0,70 \pm 0,40$ & $0,71 \pm 0,39$ & 0,8 \\
\hline Estenose em diâmetro pré-procedimento, \% & $72,9 \pm 14,5$ & $71,3 \pm 14,3$ & 0,2 \\
\hline Comprimento da lesão, mm & $10,9 \pm 6,2$ & $10,5 \pm 6,6$ & 0,4 \\
\hline Diâmetro luminal mínimo pós-procedimento, mm & $2,35 \pm 0,61$ & $2,30 \pm 0,57$ & 0,4 \\
\hline Estenose em diâmetro pós-procedimento, \% & $18,3 \pm 16,5$ & $17,0 \pm 14,2$ & 0,4 \\
\hline
\end{tabular}

Números são porcentagem ou média \pm desvio padrão. $\mathrm{n}=$ número de pacientes.

no, idosos, dialíticos e uniarteriais, e menos freqüentemente diabéticos.

Pacientes com IMC $\leq 20 \mathrm{~kg} / \mathrm{m}^{2}$ apresentavam peso, altura e IMC médios de 49,4 \pm 7,1 kg, 1,62 \pm 0,10 m, e $18,7 \pm 1,1 \mathrm{~kg} / \mathrm{m}^{2}$, respectivamente. Peso, altura e IMC médios de pacientes com IMC > $20 \mathrm{~kg} / \mathrm{m}^{2}$ foram de
$74,4 \pm 13,8 \mathrm{~kg}, 1,64 \pm 0,09 \mathrm{~m}$, e $27,3 \pm 4,3 \mathrm{~kg} / \mathrm{m}^{2}$, respectivamente ( $p<0,01$ para todas as características).

Os pacientes foram seguidos por uma mediana de 973 dias (intervalo interquartil de 545-1.326 dias). Pacientes do grupo baixo peso apresentaram mortalidade significativamente maior que a dos pacientes com 
IMC > 20 após 2,5 anos da angioplastia $(19,4 \%$ vs. $6,9 \%$, respectivamente; hazard ratio [HR]: 2,51, intervalo de confiança de 95\% [IC 95\%]: 1,61-3,91; p < 0,01) (Figura 1). Como ilustrado na Figura 1, o maior risco de óbito em pacientes do grupo baixo peso já era evidente nos trinta primeiros dias após o procedimento e manteve-se crescente ao longo de todo o período de observação.

Mesmo após o ajuste multivariado para a presença de outros fatores de alto risco (idade, diabetes melito, infarto do miocárdio prévio, insuficiência cardíaca, infarto agudo do miocárdio à admissão, cirurgia de revascularização do miocárdio prévia, doença coronariana triarterial, insuficiência renal crônica dialítica,

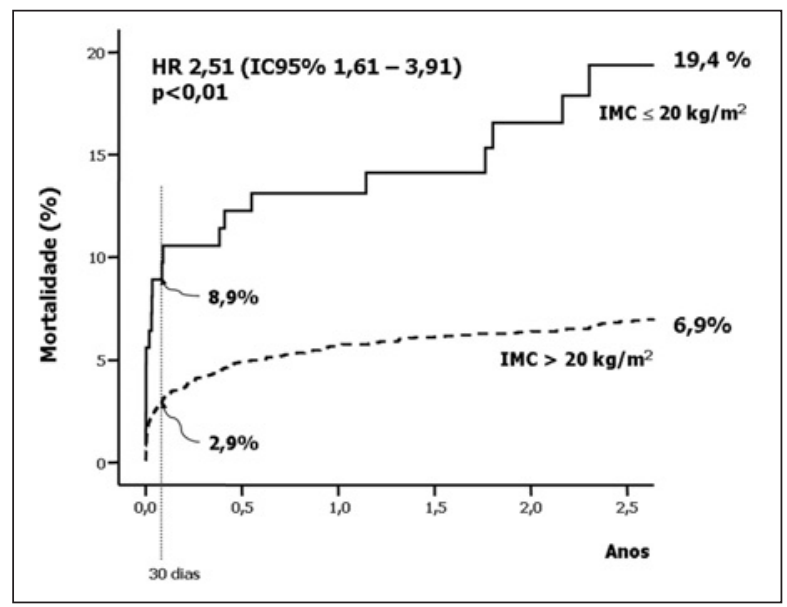

Figura 1 - Mortalidade cumulativa ao longo de 2,5 anos após angioplastia coronária de pacientes com índice de massa corporal $\leq 20 \mathrm{~kg} / \mathrm{m}^{2}$ e de pacientes com índice de massa corporal $>20 \mathrm{~kg} / \mathrm{m}^{2}$. Observa-se que pacientes com muito baixo peso apresentam mortalidade aumentada já evidente nos primeiros trinta dias e que a diferença de risco entre os grupos é crescente ao longo de todo o período de observação. $\mathrm{HR}=$ hazard ratio; IC $95 \%=$ intervalo de confiança de 95\%; IMC = índice de massa corporal. doença vascular periférica e acidente vascular cerebral prévio), a presença de $\mathrm{IMC} \leq 20 \mathrm{~kg} / \mathrm{m}^{2}$ persistiu como um fator independente associado a aumento da mortalidade em pacientes tratados com angioplastia coronária (HR: 2,04; IC 95\%: 1,28-3,25; p < 0,01) (Tabela 2; Figura 2).

\section{DISCUSSÃO}

O principal achado deste estudo é que a presença de baixo peso ponderal se associa independentemente a maiores taxas de mortalidade nos primeiros 2,5 anos após intervenção coronária percutânea. Pacientes com $\mathrm{IMC} \leq 20 \mathrm{~kg} / \mathrm{m}^{2}$ apresentaram risco de óbito aproximadamente duas vezes maior que pacientes sem essa característica, mesmo quando considerados os efeitos de outros preditores prognósticos.

Estudos prévios na população americana já haviam sugerido o efeito deletério do baixo peso na evolução a curto prazo de pacientes tratados com angioplastia coronária 4,9,10. Nossos resultados ampliam esse conceito e demonstram que pacientes com muito baixo peso persistem sob risco elevado de óbito mesmo após 2,5 anos da angioplastia. É importante notar que o valor prognóstico do baixo peso independe do quadro clínico e de outros fatores de risco clássicos.

Na presente série, pacientes com muito baixo peso representaram somente $3,4 \%$ da população total. No entanto, apesar de pouco freqüente, esse subgrupo de risco pode ser facilmente identificado na prática clínica, sem necessidade de testes adicionais. Em decorrência disso, e do impacto prognóstico dessa característica, a pesquisa do muito baixo peso pode ser utilizada como importante ferramenta de estratificação de risco para pacientes tratados com angioplastia.

Nosso estudo não permite avaliar os fatores causais relacionados ao aumento da mortalidade em pacientes com IMC $\leq 20 \mathrm{~kg} / \mathrm{m}^{2}$. Esse subgrupo apresen-

TABELA 2

Preditores multivariados de mortalidade tardia após angioplastia coronária

\begin{tabular}{lccc}
\hline Característica & Hazard ratio & Intervalo de confiança de $\mathbf{9 5 \%}$ & $\mathbf{p}$ \\
\hline Insuficiência renal crônica dialítica & 2,61 & $1,13-6,03$ & 0,03 \\
Infarto agudo do miocárdio à admissão & 2,16 & $1,67-2,75$ & $<0,01$ \\
Insuficiência cardíaca & 2,15 & $1,63-2,83$ & $<0,01$ \\
Índice de massa corporal $\leq 20 \mathrm{~kg} / \mathrm{m}^{2}$ & 2,04 & $1,28-3,25$ & $<0,01$ \\
Doença vascular periférica & 1,82 & $1,15-2,86$ & 0,01 \\
Doença coronária triarterial & 1,71 & $1,30-2,24$ & $<0,01$ \\
Diabetes melito & 1,37 & $1,04-1,70$ & 0,03 \\
Idade & 1,05 & $1,04-1,06$ & $<0,01$ \\
Infarto do miocárdio prévio & 0,99 & $0,76-1,27$ & 0,9 \\
Cirurgia de revascularização miocárdica prévia & 0,99 & $0,71-1,37$ & 1,0 \\
Acidente vascular cerebral prévio & 0,94 & $0,54-1,62$ & 0,8 \\
\hline
\end{tabular}




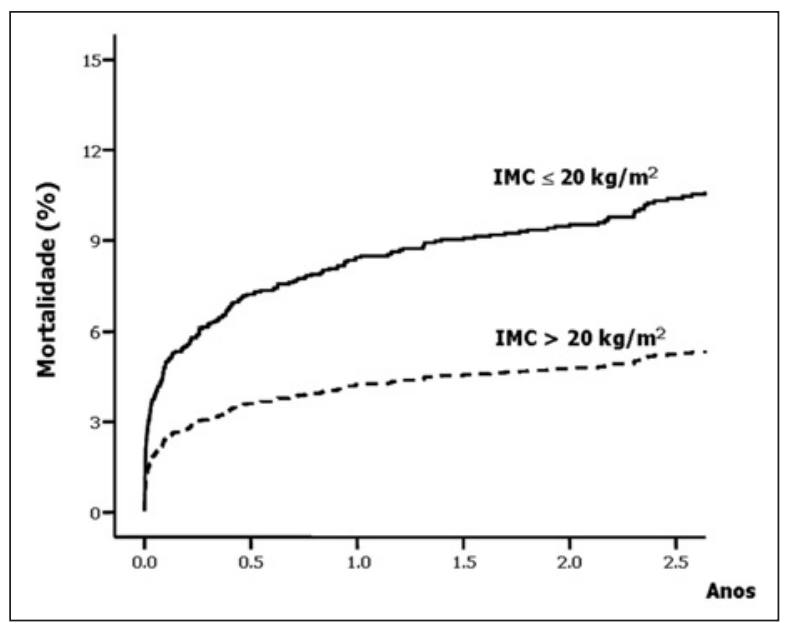

Figura 2 - Curvas de mortalidade estimada ao longo de 2,5 anos para pacientes com índice de massa corporal $\leq 20 \mathrm{~kg} / \mathrm{m}^{2}$ e pacientes com índice de massa corporal $>20 \mathrm{~kg} / \mathrm{m}^{2}$ após correção multivariada para a presença de outros fatores prognósticos. IMC = índice de massa corporal.

tou aumento do risco de óbito precoce $(8,9 \%$ vs. $2,9 \%$ aos trinta dias; diferença de mortalidade entre os grupos de $6,0 \%$ ), indicando que o baixo peso possivelmente se associa a maior risco de complicações fatais relacionadas ao procedimento. De fato, relatos prévios têm sugerido aumento da freqüência de complicações hemorrágicas e de via de acesso após angioplastia em pacientes de baixo peso ${ }^{4,9,10}$. No entanto, a persistência do risco elevado mesmo após o período periprocedimento $(19,4 \%$ vs. $6,9 \%$ aos 2,5 anos; diferença de mortalidade entre os grupos de $12,5 \%$ ) indica que outros fatores possam também influenciar o prognóstico desses pacientes. Em estudo prévio na população geral, com ou sem doença coronariana diagnosticada, o baixo peso ponderal não se associou a maior incidência de óbito por neoplasia, mas se relacionou a excesso de óbitos cardiovasculares após catorze anos de seguimento ${ }^{2}$.

\section{CONCLUSÃO}

O muito baixo peso ponderal, indicado pelo IMC $\leq 20 \mathrm{~kg} / \mathrm{m}^{2}$, é um fator de risco independente para a ocorrência de óbito precoce e tardio nos primeiros 2,5 anos após intervenção coronária percutânea. Em decorrência de seu valor prognóstico e da facilidade de sua identificação, a pesquisa do muito baixo peso pode ser amplamente utilizada como ferramenta de estratificação de risco para pacientes tratados com angioplastia coronária no dia-a-dia.

\section{REFERÊNCIAS BIBLIOGRÁFICAS}

1. Manson JE, Willett WC, Stampfer MJ, Colditz GA, Hunter D), Hankinson SE, et al. Body weight and mortality among women. N Engl J Med. 1995;333(11):677-85.

2. Calle EE, Thun MJ, Petrelli JM, Rodriguez C, Heath CW Jr. Body-mass index and mortality in a prospective cohort of U.S. adults. N Engl J Med. 1999;341(15):1097-105.

3. Gurm HS, Whitlow PL, Kip KE. The impact of body mass index on short- and long-term outcomes in patients undergoing coronary revascularization. Insights from the bypass angioplasty revascularization investigation (BARI). J Am Coll Cardiol. 2002;39(5):834-40.

4. Minutello RM, Chou ET, Hong MK, Bergman G, Parikh M, lacovone $\mathrm{F}$, et al. Impact of body mass index on in-hospital outcomes following percutaneous coronary intervention (report from the New York State Angioplasty Registry). Am J Cardiol. 2004;93(10):1229-32.

5. Mora S, Yanek LR, Moy TF, Fallin MD, Becker LC, Becker DM. Interaction of body mass index and Framingham risk score in predicting incident coronary disease in families. Circulation. 2005;111(15):1871-6.

6. Gruberg L, Mercado N, Milo S, Boersma E, Disco C, van Es GA, et al. Impact of body mass index on the outcome of patients with multivessel disease randomized to either coronary artery bypass grafting or stenting in the ARTS trial: The obesity paradox II? Am J Cardiol. 2005;95(4):439-44.

7. Eisenstein EL, McGuire DK, Bhapkar MV, Kristinsson A, Hochman JS, Kong DF, et al. Elevated body mass index and intermediate-term clinical outcomes after acute coronary syndromes. Am J Med. 2005;118(9):981-90.

8. Romero-Corral A, Montori VM, Somers VK, Korinek J, Thomas RJ, Allison TG, et al. Association of bodyweight with total mortality and with cardiovascular events in coronary artery disease: a systematic review of cohort studies. Lancet. 2006;368(9536):666-78.

9. Shubair MM, Prabhakaran P, Pavlova V, Velianou JL, Sharma AM, Natarajan MK. The relationship of body mass index to outcomes after percutaneous coronary intervention. J Interv Cardiol. 2006;19(5):388-95.

10. Ellis SG, Elliott J, Horrigan M, Raymond RE, Howell G. Low-normal or excessive body mass index: newly identified and powerful risk factors for death and other complications with percutaneous coronary intervention. Am J Cardiol. 1996;78(6):642-6.

11. Reiber JH, Serruys PW, Kooijman CJ, Wijns W, Slager CJ, Gerbrands JJ, et al. Assessment of short-, medium-, and long-term variations in arterial dimensions from computerassisted quantitation of coronary cineangiograms. Circulation. 1985;71(2):280-8.

12. Smith SC Jr, Feldman TE, Hirshfeld JW Jr, Jacobs AK, Kern MJ, King SB $3^{\text {rd }}$, et al. ACC/AHA/SCAl 2005 guideline update for percutaneous coronary intervention: a report of the American College of Cardiology/American Heart Association Task Force on Practice Guidelines (ACC/AHA/SCAI Writing Committee to Update 2001 Guidelines for Percutaneous Coronary Intervention). Circulation. 2006;113(7): e166-286. 